\title{
Are serum Netrin-4 levels predictive of preeclampsia?
}

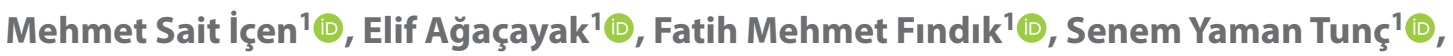

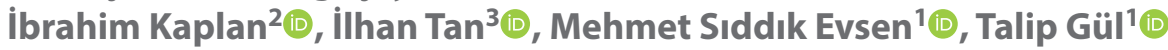 \\ ${ }^{1}$ Department of Obstetrics and Gynecology, Dicle University Medical Faculty, Diyarbakır, Turkey \\ ${ }^{2}$ Department of Biochemistry, Dicle University Medical Faculty, Diyarbakir, Turkey \\ ${ }^{3}$ Department of Pediatrics, Dicle University Medical Faculty, Diyarbakir, Turkey
}

\begin{abstract}
Objective: To investigate the levels of anti-angiogenic factors, namely sFlt-1 and Netrin-4, in patients with preeclampsia (PE). Material and methods: Cord-blood (UC) sFlt-1 and Netrin-4 concentrations were measured in 30 patients with severe PE, 30 patients with PE and 30 control infants and their mothers (MS).

Results: Maternal sFlt-1 levels were significantly higher in the severe PE and PE groups than in the control group. There were no statistical differences among the three groups in maternal and fetal Netrin-4 levels. But Netrin-4 levels were found to be the lowest in the control group and higher in the PE and severe PE groups. The correlation analysis revealed a positive correlation between maternal sFIt- 1 levels and maternal Netrin-4 levels $(p=0.012$, and $r=0.263)$, maternal sFlt- 1 levels and fetal sFlt-1 levels $(p=0.012$, and $r=0.263)$.

Conclusions: There was a positive correlation found between maternal sFlt-1 levels and maternal Netrin-4 levels. We are of the opinion that elevation in levels of Netrin- 4 might be secondary to placental hypoxia occurring in PE. The present study led to the consideration of anti-angiogenic biomarkers (sFlt-1 and Netrin-4) on automated platforms for clinical use as an aid in establishing the diagnosis and prognosis of PE.
\end{abstract}

Key words: sFlt-1; Netrin-4; preeclampsia; anti-angiogenic effects

Ginekologia Polska 2020; 91, 12: 733-739

\section{INTRODUCTION}

The exact mechanism of preeclampsia (PE) has yet to be fully unraveled; however, healthcare professionals consider PE to be a condition arising from placental insufficiency caused by insufficient remodeling of the maternal vascular bed. Angiogenetic imbalance assumes an important role in the pathophysiology of PE [1]. When angiogenic factors' functions are hindered by anti-angiogenic factors in patients with PE, hypertension, endothelial dysfunction, and proteinuria occur [2]. Despite it being a prominent feature, the presence of proteinuria is no longer considered to be the ultimate diagnostic criterion for PE. PE is defined by hypertension accompanied by one or more of the following conditions: thrombocytopenia (a platelet count lower than 100,000/microliter), impaired liver function, new-onset renal failure, pulmonary edema, and new-onset cerebral and visual disturbances [1]. It has serious potential to aggravate maternal mortality and morbidity rates in the short-, medium-, and long-term. In addition, it might bring about serious consequences for the fetus, including prenatal mortality, intrauterine growth restriction, and premature delivery [3]. The reported frequency of PE varies from 3-10\% $[4,5]$.

Vascular endothelial growth factor (VEGF) is an endothelial-specific mitogen that plays a key role in promoting angiogenesis [6]. The VEGF family has five members: VEGF-A, VEGF-B, VEGF-C, VEGF-D, and placenta growth factor (PIGF). VEGF-A binds with high affinity to fms-like tyrosine kinase-1 (FIt-1), also known as VEGFR-1. Soluble fms-like tyrosine kinase 1 (sFIt-1), a soluble form of VEGF receptor Flt-1, is anti-angiogenic [3]. sFlt-1 is significantly involved in the etiology of $\mathrm{PE}$. One study reported that the effects of VEGF on arterioles were inhibited by sFlt-1 elevation [2]. Another study reported that excess amounts of sFlt-1 released by trophoblasts contributed significantly to the development of PE [7]. One group of researchers reported that concentrations of anti-angiogenic factors, including serum-soluble fms-like tyrosine kinase 1 (sFlt-1), were elevated in patients with PE compared to healthy control subjects. In contrast, concentrations of significant angiogenic factors, including serum VEGF, were diminished 
in patients with PE compared to healthy control subjects [8]. Maternal sFlt-1 concentration is known to be elevated in patients with PE. The concentration of sFlt-1 is increased in $P E$ in direct correlation with the severity of the condition [2].

Netrins are bifunctional and assume either pro-angiogenic or anti-angiogenic roles depending on the receptor and its expression [9]. Netrins have been shown to play a key role in angiogenic processes. They are located in the placenta and are important for both placental development and placental vascular development [10]. Koch et al. [11] were the first to define Netrin-4, a member of evolutionarily conserved extracellular protein family netrins. It is also referred to as $\beta$-netrin because it is more closely related to the laminin chains than the other netrins that are known to exhibit homology to the short arms of laminin chains. It is also defined as basement membrane component found in the basement membranes of the vasculature, kidneys, and ovaries. It does not play an active role in normal vascular development; however, its expression may have a major effect on pathological blood vessel formation and increased hypoxia. Netrin-4 also plays a key role in angiogenesis and is anti-angiogenic [12]. Finally, several studies have reported that it significantly inhibits VEGF-induced angiogenesis $[13,14]$.

The purpose of the present study was to investigate the levels of anti-angiogenic factors-namely, sFlt- 1 and Netrin-4 in patients with $\mathrm{PE}$, a condition that lacks a thorough etiological and pathogenetic explanation. The results of the study might improve the prediction and treatment of PE.

\section{MATERIAL AND METHODS}

\section{Patients and study groups}

This prospective clinical study was conducted between March 2015 and February 2017 at a tertiary centre. A total of 90 patients were included in this study; 30 were severe $P E$, 30 were PE and 30 were a control group. Before the study, approval was obtained from the local ethics committee (approval no: 2015/178).

Inclusion criteria were patients ages 16 to 44 years with PE diagnosis. Diagnosis of severe PE and PE was established using the ACOG criteria [1]. Exclusion criteria included the following: additional systemic diseases, twin pregnancy, fetal abnormalities, HELLP syndrome and eclampsia. The control group consisted of the patients who delivered in our clinic without any additional disease.

Serum measurements of blood samples and anti-angiogenic biomarkers

Maternal peripheral venous blood samples were taken in the first stage of the delivery in patients undergoing normal vaginal delivery, right before the administration of anesthesia in patients undergoing $\mathrm{C}$-section under general anesthesia, and right before the administration of spinal anesthesia in patients undergoing $\mathrm{C}$-section under spinal anesthesia. Fetal cord blood samples were taken from the umbilical artery after the umbilical cord was clamped and cut. Sera were isolated by centrifugation and kept at $-80^{\circ} \mathrm{C}$ until further analysis. Hematological parameters (Hemoglobin, hematocrit and platelet) were measured by means of an automated hematology analyzer (Abbott Cell-Dyn Sapphire; Abbott Laboratory, Abbott Park, IL, USA). Levels of urea, creatinine, aspartate transaminase (AST) and alanine aminotransferase (ALT) were determined by means of an automated chemistry analyzer by the Abbott Architect C 16000 autoanalyzer (Architect C16000 auto-analyzer; Abbott Laboratory, Abbott Park, IL, USA) using commercial kits (Abbott) in the peripheral venous blood samples. Proteinuria was assessed using an automated urinalysis system by IRIS IQ 200 ELITE (Iris Diagnostics Chatsworth, CA, USA). Serum sFlt- 1 and Netrin-4 levels were determined by means of an enzyme-linked immunosorbent assay (ELISA) kit (Shanghai Sunred Biological Technology Co., Shanghai, China) according to the manufacturer's instructions. The serial numbers were 201-12-0228 and 201-12-1298, respectively. All the measurements were performed by the same investigator in a blinded manner.

\section{Statistical analysis}

All statistical analyses were performed by SPSS-21. Data were expressed as mean $\pm \mathrm{sd}$. According to our study results for sFlt-1 and Netrin-4 levels, sample size of the study population was calculated to be 30 patients per group ( $a=0.05$ and the study power $=80 \%$ ). According to distribution assumptions for the continuous variables, the categorical data are given using numbers and percentages. The distribution of the data was evaluated using the Kolmogorov-Smirnov test. Group comparisons were performed using the Mann-Whitney $U$ test. Correlations were tested using the Spearman correlation analysis. A p value smaller than 0.05 was considered statistically significant.

\section{RESULTS}

No significant differences were observed among the three groups in terms of age or parity. Gestational age, fetal weight, and one- and five-minute Apgar scores were significantly lower in the severe PE group compared to the $\mathrm{PE}$ and control groups. There were significant differences among the three groups in terms of systolic and diastolic blood pressure (Tab. 1). $\mathrm{Hb}$ and $\mathrm{Htc}$ levels were significantly higher in the severe PE group than they were in the control group. Urea and creatinine levels were significantly higher in the severe PE and PE groups than they were in the control group. ALT and AST levels were significantly higher in the severe PE group than in the PE and control groups. Mater- 


\begin{tabular}{|c|c|c|c|c|}
\hline & Control $(n=30)$ & Preeclampsia $(n=30)$ & Severe preeclampsia $(n=30)$ & p \\
\hline Maternal age (years) & $30.10 \pm 5.30$ & $30.33 \pm 7.11$ & $30.53 \pm 30.77$ & $\begin{array}{l}\alpha: 0.807 \beta: \\
0.756 \\
€: 0.941\end{array}$ \\
\hline Parity & $2.23 \pm 1.75$ & $2.23 \pm 2.70$ & $2.13 \pm 3.07$ & $\begin{array}{l}\alpha: 0.464 \beta: \\
0.248 \\
€: 0.539\end{array}$ \\
\hline Gestational age (weeks) & $36.37 \pm 2.52$ & $36.37 \pm 2.54$ & $34.43 \pm 3.09$ & $\begin{array}{l}\alpha: 0.009 \beta: \\
0.010 \\
€: 0.970\end{array}$ \\
\hline Systolic blood pressure [mm Hg] & $117.33 \pm 11.72$ & $144.67 \pm 5.07$ & $172.33 \pm 18.51$ & $\begin{array}{l}\alpha: 0.000 \beta: \\
0.000 \\
€: 0.000\end{array}$ \\
\hline Diastolic blood pressure [mm Hg] & $74.33 \pm 7.73$ & $91.00 \pm 7.58$ & $172.33 \pm 18.51$ & $\begin{array}{l}\alpha: 0.000 \beta: \\
0.000 \\
€: 0.000\end{array}$ \\
\hline Birth weight [g] & $2827.17 \pm 620.75$ & $2777.30 \pm 637.82$ & $2293.17 \pm 813.89$ & $\begin{array}{l}\alpha: 0.008 \beta: \\
0.004 \\
€: 0.923\end{array}$ \\
\hline 1-min Apgar score & $6.80 \pm 1.49$ & $6.27 \pm 1.14$ & $5.47 \pm 1.33$ & $\begin{array}{l}\alpha: 0.027 \beta: \\
0.001 \\
€: 0.075\end{array}$ \\
\hline 5-min Apgar score & $8.47 \pm 1.04$ & $8.23 \pm 0.97$ & $7.57 \pm 1.13$ & $\begin{array}{l}\alpha: 0.021 \beta: \\
0.003 \\
€: 0.360\end{array}$ \\
\hline
\end{tabular}

a - comparison of severe and preeclampsia; $\beta$ - comparison of severe preeclampsia and the control group; $€$ - comparison of preeclampsia and control group; values shown are means \pm SEM

nal sFlt-1 levels were significantly higher in the severe PE and PE groups than they were in the control group. However, no significant difference was found between maternal sFlt- 1 values in the severe PE versus PE groups. In contrast, fetal sFlt-1 levels were significantly higher in the severe PE group than they were in the control group. There were no statistically significant differences among the three groups in terms of maternal and fetal Netrin-4 levels. However, maternal and fetal Netrin-4 levels were lowest in the control group and higher in the PE and severe PE groups (Tab. 2). In all three groups, fetal Netrin-4 levels were higher than maternal Netrin-4 levels. The correlation analysis revealed a positive correlation between maternal sFlt-1 levels and maternal urea levels $\left(p=0.010, r=0.269^{*}\right)$ (Fig. $\left.1 \mathrm{~A}\right)$ as well as a negative correlation between maternal sFlt-1 levels and maternal platelet counts ( $p=0.040, r=-0.217^{*}$ ) (Fig. 1B). The correlation analysis revealed a positive correlation between maternal sFlt-1 levels and maternal Netrin-4 levels $\left(p=0.012, r=0.263^{*}\right)$ (Fig. 1C). Lastly, there was a positive correlation between maternal sFlt-1 levels and fetal sFlt-1 levels ( $\left.p=0.012, r=0.263^{*}\right)$ (Fig. 1D).

\section{DISCUSSION}

This study investigated levels of anti-angiogenic biomarkers (sFlt-1 and Netrin-4) in the diagnosis of PE. Maternal
sFlt-1 and Netrin-4 levels were higher in the severe PE and PE groups than they were in the control group. Further, there was a positive correlation between maternal sFlt-1 levels and maternal Netrin-4 levels.

For patients with $\mathrm{PE}$, the earlier the gestational week, the higher the chances of maternal and prenatal mortality and morbidity. There is a known association between abnormal placentation and $P E$, which is thought to arise from insufficient trophoblast invasion in the maternal spiral arteries in the early stages of gestation [15]. It is for this reason that generally, gestation is at an earlier stage and fetal weight is lower when patients develop severe PE. In the present study, fetal weight was lower in the severe PE group than it was in the PE and control groups. Gestation was also at an earlier stage in the severe PE group than it was in the PE and control groups. However, there was no correlation between fetal and maternal sFlt-1 levels and gestational week.

Catherine et al. investigated pregnancies complicated by $P E$ and reported higher sFlt-1 levels in both maternal and fetal sera compared to control sera. Elevated levels in the fetal sera were limited compared to levels in the maternal sera, but they were still considered to be high. In this respect, there was a positive correlation between maternal and fetal sFlt-1 levels in patients with PE. The researchers found that maternal sFlt-1 levels were 29 times higher than fetal 


\begin{tabular}{|c|c|c|c|c|}
\hline & Control $(n=30)$ & Preeclampsia $(n=30)$ & Severe preeclampsia $(n=30)$ & p \\
\hline Haemoglobin [g/dL] & $11.85 \pm 1.91$ & $11.85 \pm 1.48$ & $12.84 \pm 1.81$ & $\begin{array}{l}\text { a: } 0.011 \\
\beta: 0.018 \\
€: 0.807\end{array}$ \\
\hline Hematocrit (\%) & $36.84 \pm 4.99$ & $37.89 \pm 3.91$ & $39.49 \pm 5.47$ & $\begin{array}{l}\alpha: 0.183 \\
\beta: 0.061 \\
€: 0.473\end{array}$ \\
\hline Platelet count $\left[\mathrm{mm}^{3}\right]$ & $259.47 \pm 72.45$ & $273.40 \pm 59.87$ & $219.27 \pm 59.92$ & $\begin{array}{l}\alpha: 0.001 \\
\beta: 0.059 \\
€: 0.290\end{array}$ \\
\hline Urea [mg/dL] & $17.77 \pm 5.15$ & $22.33 \pm 6.61$ & $23.63 \pm 7.92$ & $\begin{array}{l}\alpha: 0.615 \\
\beta: 0.000 \\
€: 0.000\end{array}$ \\
\hline Creatinine $[\mathrm{mg} / \mathrm{dL}]$ & $0.54 \pm 0.06$ & $2.73 \pm 11.57$ & $0.62 \pm 0.07$ & $\begin{array}{l}\alpha: 0.695 \\
\beta: 0.000 \\
€: 0.000\end{array}$ \\
\hline ALT [U/L] & $12.20 \pm 3.50$ & $12.77 \pm 5.74$ & $25.23 \pm 27.36$ & $\begin{array}{l}\alpha: 0.028 \\
\beta: 0.026 \\
€: 0.841\end{array}$ \\
\hline AST [U/L] & $19.13 \pm 5.51$ & $21.60 \pm 7.64$ & $33.90 \pm 32.89$ & $\begin{array}{l}\alpha: 0.020 \\
\beta: 0.001 \\
€: 0.147\end{array}$ \\
\hline Maternal Netrin- 4 [pg/mL] & $177.00 \pm 71.76$ & $192.67 \pm 68.09$ & $224.47 \pm 182.50$ & $\begin{array}{l}\alpha: 0.836 \\
\beta: 0.442 \\
€: 0.487\end{array}$ \\
\hline Fetal Netrin-4 [pg/mL] & $264.93 \pm 120.86$ & $295.40 \pm 240.31$ & $359.37 \pm 282.47$ & $\begin{array}{l}\alpha: 0.089 \\
\beta: 0.730 \\
€: 0.110\end{array}$ \\
\hline Maternal sFlt-1 [pg/mL] & $4220.97 \pm 1804.13$ & $7184.47 \pm 6934.28$ & $8363.40 \pm 5303.40$ & $\begin{array}{l}\alpha: 0.061 \\
\beta: 0.000 \\
€: 0.010\end{array}$ \\
\hline Fetal sFlt-1 [pg/mL] & $423.37 \pm 172.75$ & $509.60 \pm 136.97$ & $570.63 \pm 106.13$ & $\begin{array}{l}\alpha: 0.243 \\
\beta: 0.001 \\
€: 0.061\end{array}$ \\
\hline
\end{tabular}

a - comparison of severe and preeclampsia; $\beta$ - comparison of severe preeclampsia and the control group; $€$ - comparison of preeclampsia and control group; values shown are means \pm SEM

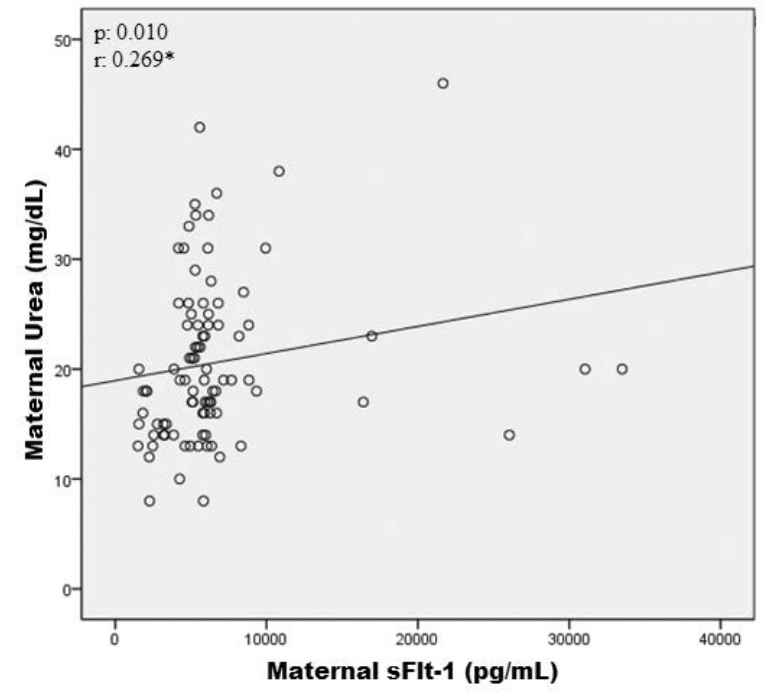

Figure 1A. Correlation analysis between maternal sFlt-1 levels and maternal urea levels

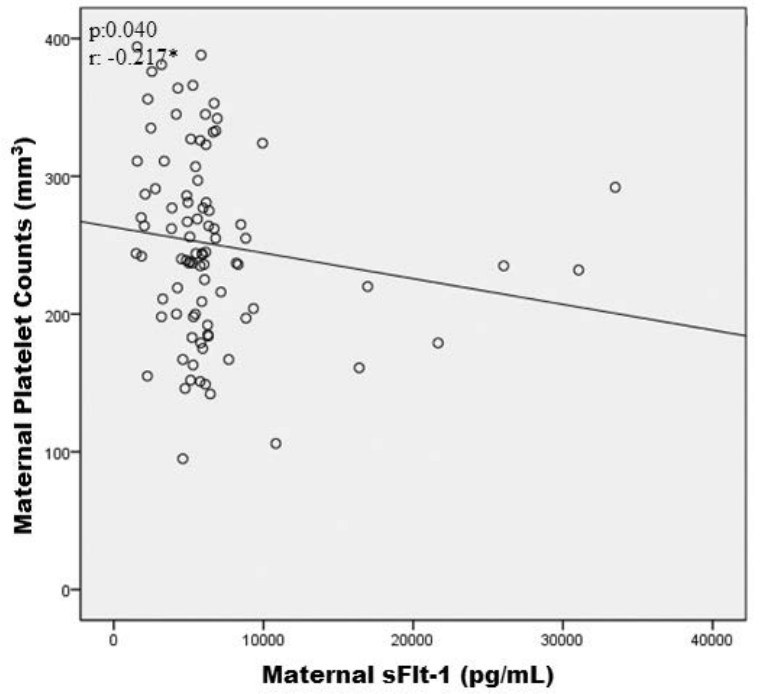

Figure 1B. Correlation analysis between maternal sFlt-1 levels and maternal platelet counts 


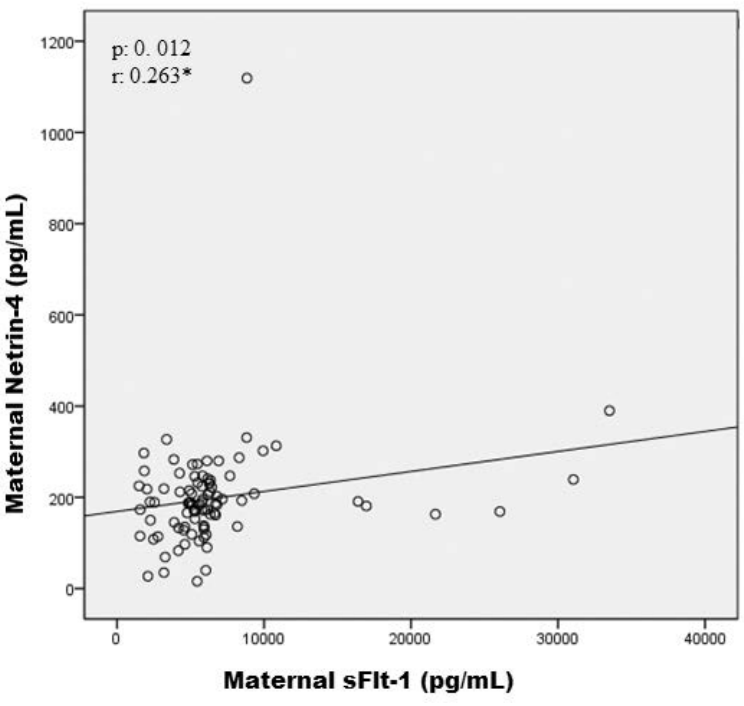

Figure 1C. Correlation analysis between maternal sFlt-1 levels and maternal Netrin-4 levels

sFlt-1 levels. This specific study observed that sFlt-1 elevation in PE was not sourced from fetal sFlt-1 levels; however, sFlt-1 elevation was higher in severe PE patients compared to mild PE patients, and maternal sFlt-1 elevation correlated with the severity of the condition [16]. Similarly, a study evaluating late-onset $P E$ reported that the level of maternal sFlt-1 in the PE group was significantly higher than it was in the control group, but unlike this, height was not proportional to the severity of the condition [17]. In the present study, maternal sFlt-1 levels were two times higher, whereas fetal sFlt-1 levels were 1.5 times higher, than the respective levels in the control group. In addition, there was a positive correlation between maternal and fetal sFlt-1 levels. In all three groups, maternal sFlt-1 levels were higher than fetal sFlt-1 levels (10 times higher in the control group, 14 times higher in the PE group, and 16 times higher in the severe PE group), indicating the absence of a significant association between elevation in maternal sFlt-1 levels and fetal sFlt-1 levels. In the present study, both maternal sFlt-1 and fetal sFlt-1 levels were significantly higher in the severe PE and PE groups than they were in the control group. In addition, sFlt-1 levels were higher in the severe PE group than they were in the PE group. However, the difference between the groups was not statistically significant, and no significant difference was found between the severity of the disease and the level of sFlt-1. This could be attributed to the small sample size of the study.

Several studies have reported the involvement of placental insufficiency in the etiopathogenesis of PE. The clinical manifestation is marked by placental hypoxia; hypoperfusion, hypoxia, and ischemia are critical components of the pathogenesis of PE. Hypoperfusion appears to be

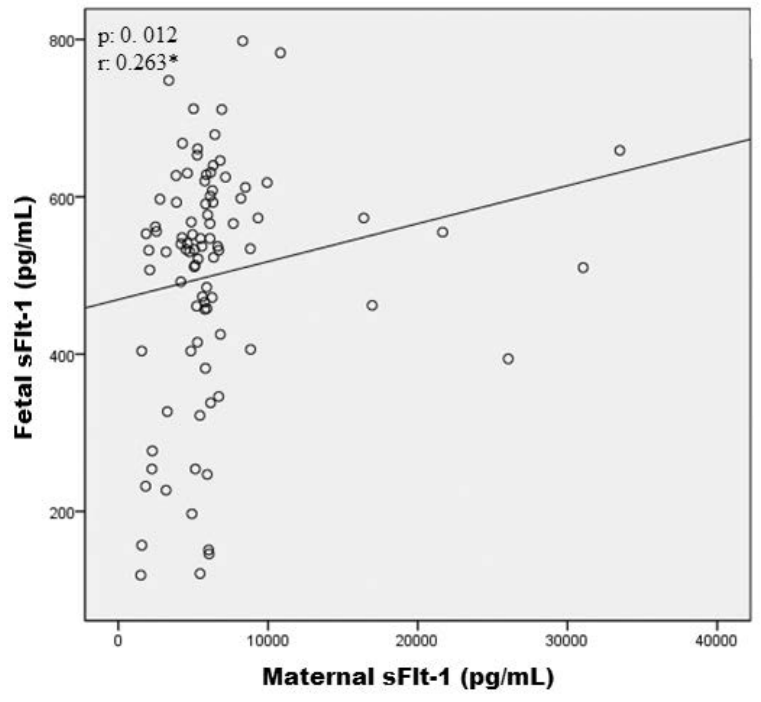

Figure 1D. Correlation analysis between maternal sFlt-1 levels and fetal sFlt-1 levels

both a cause and a consequence of abnormal placental development. In response to placental hypoxia, placenta produces pathogenic factors to be released into the maternal circulation. These factors disrupt maternal endothelial cell functions and cause signs and symptoms of PE. Previous studies reported that anti-angiogenic factors were increased in PE. One of these factors, sFlt-1, was involved in the pathogenesis of PE $[2,16,18]$. One study reported increased Netrin-4 release by Müllerian cells in hypoxic patients. Netrin-4 stimulates angiogenesis through the deleted in colorectal cancer (DCC) receptor; it was found to be highly anti-angiogenic [19]. It was further reported to exhibit a dose-dependent inhibitory effect on cellular proliferation and assume anti-angiogenic roles in human placenta [20]. In agreement with the literature, in the present study, sFlt-1 was high in the PE group and even higher in the severe $P E$ group. Further, there was a positive correlation between maternal sFlt- 1 levels and maternal Netrin-4 levels. The present authors opine that elevation in Netrin-4 levels might be secondary to placental hypoxia occurring in PE. Moreover, that elevation Netrin-4 levels is correlated with elevation in sFlt-1 levels, an anti-angiogenic agent, indicates that Netrin-4 might assume an anti-angiogenic role in conditions such as PE. This finding supports previous findings in the literature.

When sFlt-1 levels are elevated in the maternal circulation, free VEGF levels ensuring the continuity of angiogenetic and endothelial functions drop accordingly. Similarly, increased Netrin-4 levels decrease VEGF levels [21]. In the present study, maternal Netrin-4 levels were positively correlated with maternal sFlt-1 levels. This suggests that these two anti-angiogenic factors concurrently increase due to the 
decreasing pro-angiogenic factors in PE; further, it suggests that Netrin-4 is an anti-angiogenic factor in PE.

VEGF itself and VEGFR play roles not only in physiological but also in pathologic angiogenesis [7]. The overexpression of Netrin-4 has been reported to affect VEGF/basic fibroblast growth factor-induced angiogenesis [13]. In a previous study, Netrin-4 was shown to have anti-angiogenic features in high levels and be present in vasculature, kidneys, and ovaries [11]. It was further reported to stimulate endothelial cell activities related to angiogenesis. In addition, it acted as an anti-angiogenic agent when present in high levels; these levels tended to increase secondary to hypoxia [22]. In a study by Boutsikou et al. that investigated intrauterine growth restriction (IUGR), which is characterized by abnormal feto-placental angiogenesis, maternal Netrin-4 levels were lower than fetal Netrin-4 levels in all of the study groups. The authors suggested that elevation in these levels was an indicator of hypoxia. Also, as the severity of IUGR increases, the level of maternal Netrin- 4 is found to be high [9]. Hypoxia has been found to increase Netrin-4 mRNA expression [19]. In the present study, maternal Netrin-4 levels were lower than fetal Netrin-4 levels; this is in accordance with the literature. Although no statistically significant difference was detected, maternal and fetal Netrin-4 levels were lowest in the control group and higher in the PE and severe PE groups - hypoxia is known to play a role in this pathogenesis. Also, as the severity of the disease increased, Netrin-4 was also present in higher levels. Based on this study's observation that elevation in Netrin-4 levels was secondary to hypoxia, and that such elevation inhibited the expression of VEGF, which is involved in angiogenesis, the authors propose that Netrin-4 might also be involved in the etiology of PE. The positive correlation between sFlt-1 and Netrin-4 also supports this hypothesis.

The small sample size was a limitation of the present study. However, its prospective design and correlation analysis make the study strong.

\section{CONCLUSIONS}

In the last decade, the molecular mechanisms of PE have been explained. Anti-angiogenic factors, such as sFlt-1, are known to play an important role in the pathogenesis of $\mathrm{PE}$ and be significantly associated with negative outcomes associated with PE. This study found a positive correlation between maternal sFlt- 1 and Netrin-4 levels. Elevation in levels of Netrin- 4 might be secondary to placental hypoxia within PE. The present study contributes to practice by urging healthcare practitioners to consider the possibility of using anti-angiogenic biomarkers (sFlt-1 and Netrin-4) on automated platforms for clinical use to aid in establishing the diagnosis and prognosis of PE. Further studies are needed to evaluate the effect of vaginal delivery or cesarean delivery on Netrin-4 levels.

\section{Conflict of interest}

We declare that we have no conflict of interest.

\section{REFERENCES}

1. American College of Obstetricians and Gynecologists, Task Force on Hypertension in Pregnancy. Hypertension in pregnancy. Report of the American College of Obstetricians and Gynecologists'Task Force on Hypertension in Pregnancy. Obstet Gynecol. 2013; 122(5): 1122-1131, doi: 10.1097/01.AOG.0000437382.03963.88, indexed in Pubmed: 24150027.

2. Maynard SE, Min JY, Merchan J, et al. Excess placental soluble fms-like tyrosine kinase 1 (sFlt1) may contribute to endothelial dysfunction, hypertension, and proteinuria in preeclampsia. J Clin Invest. 2003; 111(5): 649-658, doi: 10.1172/JCl17189, indexed in Pubmed: 12618519.

3. Sibai B, Dekker G, Kupferminc M. Pre-eclampsia. The Lancet. 2005; 365(9461): 785-799, doi: 10.1016/s0140-6736(05)17987-2, indexed in Pubmed: 15733721

4. Maynard SE, Karumanchi SA. Angiogenic factors and preeclampsia. Semin Nephrol. 2011; 31(1): 33-46, doi: 10.1016/j.semnephrol.2010.10.004, indexed in Pubmed: 21266263.

5. Kwiatkowski S, Kwiatkowska E, Torbe A. The role of disordered angiogenesis tissue markers (sflt-1, Plgf) in present day diagnosis of preeclampsia. Ginekol Pol. 2019; 90(3): 173-176, doi: 10.5603/GP.2019.0030, indexed in Pubmed: 30950008.

6. Ferrara N, Davis-Smyth T. The biology of vascular endothelial growth factor. Endocr Rev. 1997; 18(1): 4-25, doi: 10.1210/edrv.18.1.0287, indexed in Pubmed: 9034784.

7. Shibuya M. VEGF-VEGFR Signals in Health and Disease. Biomol Ther (Seoul). 2014; 22(1): 1-9, doi: 10.4062/biomolther.2013.113, indexed in Pubmed: 24596615.

8. Levine RJ, Maynard SE, Qian C, et al. Circulating angiogenic factors and the risk of preeclampsia. N Engl J Med. 2004; 350(7): 672-683, doi: 10.1056/NEJMoa031884, indexed in Pubmed: 14764923.

9. Boutsikou T, Giotaki M, Gourgiotis D, et al. Cord blood netrin- 1 and -4 concentrations in term pregnancies with normal, restricted and increased fetal growth. J Matern Fetal Neonatal Med. 2014; 27(18): 1849-1853, doi: 10.3109/14767058.2014.905530, indexed in Pubmed: 24716747.

10. Dakouane-Giudicelli $M$, Alfaidy N, de Mazancourt P. Netrins and their roles in placental angiogenesis. Biomed Res Int. 2014; 2014: 901941, doi: 10.1155/2014/901941, indexed in Pubmed: 25143950.

11. Koch M, Murrell JR, Hunter DD, et al. A novel member of the netrin family, beta-netrin, shares homology with the beta chain of laminin: identification, expression, and functional characterization. J Cell Biol. 2000; 151(2): 221-234, doi: 10.1083/jcb.151.2.221, indexed in Pubmed: 11038171.

12. Kociok N, Crespo-Garcia S, Liang Y, et al. Lack of netrin-4 modulates pathologic neovascularization in the eye. Sci Rep. 2016; 6: 18828, doi: 10.1038/srep 18828, indexed in Pubmed: 26732856.

13. Eveno C, Broqueres-You D, Feron JG, et al. Netrin-4 delays colorectal cancer carcinomatosis by inhibiting tumor angiogenesis. Am J Pathol. 2011; 178(4): 1861-1869, doi: 10.1016/j.ajpath.2010.12.019, indexed in Pubmed: 21406174.

14. Han Y, Shao Yi, Liu T, et al. Therapeutic effects of topical netrin- 4 inhibits corneal neovascularization in alkali-burn rats. PLoS One. 2015; 10(4): e0122951, doi: 10.1371/journal.pone.0122951, indexed in Pubmed: 25853509.

15. Polliotti B. Second-trimester maternal serum placental growth factor and vascular endothelial growth factor for predicting severe, early-onset preeclampsia. Obstetrics \& Gynecology. 2003; 101 (6): 1266-1274, doi: 10.1016/s0029-7844(03)00338-7.

16. Staff AC, Braekke K, Harsem NK, et al. Circulating concentrations of sFlt1 (soluble fms-like tyrosine kinase 1) in fetal and maternal serum during pre-eclampsia. Eur J Obstet Gynecol Reprod Biol. 2005; 122(1): 33-39, doi: 10.1016/j.ejogrb.2004.11.015, indexed in Pubmed: 15935542.

17. Cim N, Kurdoglu M, Ege S, et al. An analysis on the roles of angiogenesis-related factors including serum vitamin $D$, soluble endoglin (sEng), soluble fms-like tyrosine kinase 1 (sFlt1), and vascular endothelial growth factor (VEGF) in the diagnosis and severity of late-onset preeclampsia. J Matern Fetal Neonatal Med. 2017; 30(13): 1602-1607, doi: 10.1080/14767058.2016.1219986, indexed in Pubmed: 27658884. 
18. Liu Y, Zhao Y, Yu A, et al. Diagnostic accuracy of the soluble Fms-like tyrosine kinase-1/placental growth factor ratio for preeclampsia: a meta-analysis based on 20 studies. Arch Gynecol Obstet. 2015; 292(3):507-518, doi: 10.1007/s00404-015-3671-8, indexed in Pubmed: 25716670.

19. Lange J, Yafai $Y$, Noack A, et al. The axon guidance molecule Netrin-4 is expressed by Müller cells and contributes to angiogenesis in the retina. Glia. 2012; 60(10): 1567-1578, doi: 10.1002/glia.22376, indexed in Pubmed: 22777897.

20. Dakouane-Giudicelli M, Brouillet S, Traboulsi W, et al. Inhibition of human placental endothelial cell proliferation and angiogenesis by netrin-4. Placenta. 2015; 36(11): 1260-1265, doi: 10.1016/j.placenta.2015.09.007, indexed in Pubmed: 26390805.

21. Cao B, Meng X, Fu Y, et al. Neuron-derived netrin- 1 and netrin- 4 protein are additional effective targets in diabetic retinopathy beyond VEGF. Int J Clin Exp Pathol. 2017;10(8):8174-86. PubMed PMID: 31966669; PubMed Central PMCID: PMC6965419.

22. Nacht M, St Martin TB, Byrne A, et al. Netrin-4 regulates angiogenic responses and tumor cell growth. Exp Cell Res. 2009; 315(5): 784-794 doi: 10.1016/j.yexcr.2008.11.018, indexed in Pubmed: 19094984. 